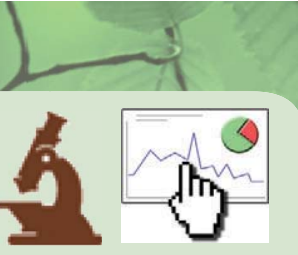

Tree Physiology 41, 1701-1713

https://doi.org/10.1093/treephys/tpab031

Research paper

\title{
Cork cells in cork oak periderms undergo programmed cell death and proanthocyanidin deposition
}

\author{
Vera Inácio ${ }^{1,2,5}$, Carolina Lobato ${ }^{1,3}$, José Graça ${ }^{4}$ and Leonor Morais-Cecílio 1
}

\begin{abstract}
${ }^{1}$ Linking Landscape, Environment, Agriculture and Food (LEAF), Institute of Agronomy, University of Lisbon, Tapada da Ajuda, 1349-017 Lisboa, Portugal; ${ }^{2}$ BiolSI_Biosystems and Integrative Sciences Institute, Faculty of Sciences, University of Lisbon, Campo Grande 016, 1749-016 Lisboa, Portugal; ${ }^{3}$ Institute of Environmental Biotechnology (UBT), Graz University of Technology, Petersgasse 12/I, 8010 Graz, Styria, Austria; ${ }^{4}$ Forest Research Center (CEF), Institute of Agronomy, University of Lisbon, Tapada da Ajuda, 1349-017 Lisboa, Portugal; ${ }^{5}$ Corresponding author (vlinacio@fc.ul.pt)
\end{abstract}

Received July 27, 2020; accepted February 15, 2021; handling Editor Christophe Plomion

Vascular plants with secondary growth develop a periderm mostly composed of dead suberized cork cells to face environmental hostile conditions. Cork oak has a highly active and long-living phellogen forming a remarkably thick periderm that is periodically debarked for industrial purposes. This wounding originates the quick formation of a new traumatic periderm, making cork oak an exceptional model to study the first periderm differentiation during normal development in young sprigs and traumatic (wound) periderm formation after debarking. Here, we studied the poorly known first periderm differentiation steps that involve cell wall suberization, polyphenolic accumulation and programmed cell death (PCD) by combining transmission electron microscopy, histochemical and molecular methods in periderms from young sprigs. These processes were further compared with traumatic periderms formed after wounding using molecular and histochemical techniques, such as the polyphenolic accumulation. In the first periderms from young sprigs, four distinct differentiation stages were defined according to the presence of PCD morphological features. First young and traumatic periderms showed an upregulation of genes related to suberin biosynthesis, proanthocyanidins biosynthesis and transport, autophagy, and PCD. Traumatic periderms revealed an overall upregulation of these genes, likely resulting from ontogeny differences and distinct phellogen origin associated with a faster metabolism, highlighting the impact of wounding on phellogen activity after debarking. First periderms from young sprigs showed gradual accumulation of proanthocyanidins in the vacuoles throughout PCD stages until total filled lumens, whereas in traumatic periderms, these compounds were found cell wall linked in already empty cells. This work enabled a comprehensive overview of the cork cells differentiation processes contributing to deepening the knowledge of the fundamental ontogenic program of this protective tissue, which is also a unique forest product, constituting the basis of a sustainable and profitable industry.

Keywordsautophagy, condensed tannins, cork, developmental programmed cell death, phellogen, Quercus suber.

\section{Introduction}

To capture sunlight, vascular plants like trees have adapted by developing lignified stems that rise above other plants. These secondary growth stems are built from two meristems: the vascular cambium and the cork cambium or phellogen (Evert 2006). The vascular cambium gives rise to xylem and phloem, the vascular tissues, whereas the phellogen produces the periderm, the tissue that surrounds the former, supplying protection against pathogens and adverse environmental conditions (Evert 2006). The periderm is formed by the phellogen, a single-layered meristem, the multi-cell layered phellem it divides outwards and the single-layered phelloderm it produces inwards (Evert 2006). In most trees, the periderm is dominantly made of phellem cells, commonly named 'cork'.

Some trees and shrubs worldwide, particularly in dry, fireridden ecosystems, have very thick periderms, making the socalled 'corky' barks. An exceptional case is found in cork oak (Quercus suber L.), a tree that grows in the southwestern Europe

(c) The Author(s) 2021. Published by Oxford University Press. All rights reserved. For permissions, please e-mail: journals.permission@oup.com 
and the northwestern tip of Africa. Due to its highly active and long-living phellogen, it forms an exceptionally thick periderm that is exploited as industrial cork (Graça 2015). Once the first cork is harvested, the torn phellogen is destroyed and reforms in the underlying phloem living cells as a traumatic phellogen. First and young periderms, which can be seen in very young sprigs, are the product of the original phellogen, which will grow to virgin cork, while the ensuing, the amadia corks, are traumatic, also known as wound periderm, the product of the traumatic phellogens. To fulfill their protective role, cork cells have 'suberized' cell walls rich in suberin, a biopolymer typical of this tissue (reviewed in Graça 2015). Several enzymes involved in suberin monomer biosynthesis, assembly and regulation have been described (reviewed in Vishwanath et al. 2015).

The first cork cells formed in very young cork oak sprigs are enriched in brown inclusions referred to as tannins (Graça and Pereira 2004). Tannins are secondary metabolites found in plant cells from several tissues and organs chemically classified as proanthocyanidins (or condensed tannins) or hydrolysable tannins (reviewed in Hassanpour et al. 2011). Proanthocyanidins (PA) are present for instance in leaf parenchyma cells of tea plants, in grape skins and barks, accumulating essentially within the vacuoles and playing essential roles in plant defense (reviewed in Hassanpour et al. 2011). Both PA and hydrolysable tannins have been found in the cork of $Q$. suber (Conde et al. 1998, Pinheiro et al. 2019).

The biosynthesis of PA building blocks is accomplished by three enzymes: leucoanthocyanidin reductase, leucoanthocyanidin dioxygenase/anthocyanidin synthase and anthocyanidin reductase, encoded by $L A R, \angle D O X / A N S$ and BAN/ANR genes, respectively (reviewed in Saito et al. 2013). The vacuolar transport of condensed tannins is accomplished by membrane transporters like the tonoplast multidrug and toxic compound extrusion (MATE) transporter previously identified in Arabidopsis thaliana, Medicago truncatula, Vitis vinifera and in Malus $x$ domestica Borkh. (reviewed in Takanashi et al. 2014) or by tannosome shuttles formed in the chloroplast (Brillouet 2014).

Another characteristic of periderm cork cells is that they are dead when they reach functional maturity, which implies that they go through a programmed cell death (PCD) process in the later stages of their differentiation. Developmental programmed cell death $(\mathrm{dPCD})$ can be induced either by differentiation as in xylem tracheary elements (xTE) or tapetum cells in anthers, or by aging such as in leaf senescence (reviewed in (Daneva et al. 2016). These processes have been distinguished based on function, occurrence, morphological and biochemical features as autolytic or non-autolytic (van Doorn 2011), and apoptoticlike PCD (reviewed in Dickman et al. 2017). The PCD execution in animals is accomplished by the cysteine proteases caspases; however, plant genomes lack truly identifiable caspases (Bonneau et al. 2008). Instead, plants possess caspase-like enzymes whose involvement in PCD processes has been identified (reviewed in Rantong and Gunawardena 2015). In addition to these caspase-like enzymes, the proteasome is also involved in plant PCD by degrading ubiquitinated proteins (reviewed in Rantong and Gunawardena 2015). Recently, a conserved core of $\mathrm{dPCD}$-associated genes encoding either proteases or nucleases was found, including, for instance, the aspartic proteinase A3 (APA3, also known as PASPA3) and bifunctional nuclease I (BFN1, also known as ENDO1, endonuclease 1; Olvera-Carrillo et al. 2015). Along with or in addition to these hydrolytic enzymes, cell contents can be removed by autophagy (reviewed in Bozhkov 2018) and related genes have been shown to be involved in APCD (Courtois-Moreau et al. 2009, Kwon et al. 2010b).

Periderm ontogeny and phellogen activity have been studied in trees with short-living periderms where the first periderm is replaced by sequent ones after several phellogen deaths (Waisel et al. 1967, Arzee et al. 1968, 1970, 1977, Mogensen 1968, Biggs and Northover 1985, Biggs 1986, Rittinger et al. 1987, Oven et al. 1999, Gričar et al. 2015). However, studies on trees with a long-living phellogen that retain a continuous periderm throughout their entire life are scarce (Graça and Pereira 2004, Shibui and Sano 2018, Alonso-Serra et al. 2019).

Studies of the cork differentiation process at the subcellular level have never been attempted in cork oak, a periderm model plant. Several transcriptomics studies in cork oak and potato have disclosed the first breakthroughs into its periderm formation (Soler et al. 2007, Pereira-Leal et al. 2014, Boher et al. 2018, Capote et al. 2018); however, the polyphenolic accumulations and PCD in these cells were never been addressed.

In this work, we studied the formation of cork oak periderms from the first phellogen derivative cell layer to the well-developed isolating tissue by analyzing the various differentiation processes involved: cell wall suberization, phenolic tannin inclusions, and finally, the PCD leading to the ultimate differentiation stage. For this purpose, the major cellular and subcellular features occurring during this process were analyzed in first periderms from young sprigs involving a combination of ultrastructural and histochemical studies. The tannin accumulation was compared in first (young) and traumatic periderms, formed after debarking, at the histological level. In parallel, this process was compared between first young and traumatic periderms by evaluating the relative expression of genes involved in the biosynthesis of suberin and PA, PCD and autophagy.

\section{Materials and methods}

\section{Sampling}

At least 10 young 1-, 2- and 3-year-old sprigs were harvested from eight randomly chosen cork oak adult trees located in 
Tapada da Ajuda field, Lisbon. Whole sprigs were harvested for the histochemical and transmission electron microscopy analyses while peeled cork (phellem) tissue was used for the gene expression studies. Amadia cork planks were extracted as described elsewhere (Inácio et al. 2018) from 12 adult trees located at a cork oak stand (montado) in Herdade dos Leitões, Montargil, Portugal and at Tapada da Ajuda. Samples of phellogen and differentiating cork cells were collected by scraping the inner surface of amadia cork planks, and small pieces from the same planks were also cut for histological analysis (Inácio et al. 2018). To simplify, hereafter, we will refer to the samples collected from young sprigs as young differentiating cork (YDC) and samples collected from amadia cork planks as traumatic differentiating cork (TDC). All tissues were harvested during the period of intense phellogen activity (July 2016) and immediately fixed or stored in liquid nitrogen until further use. The absence of other tissues besides phellem was confirmed by fluorescence microscopy in detached phellem peel and small pieces cut from the inner surface of cork planks (see Figures S1 and S2 available as Supplementary data at Tree Physiology Online).

\section{Fixation and sectioning}

Immediately after collection, the peeled periderms and whole sprigs were fixed in $4 \%$ paraformaldehyde in $1 \times$ (phosphatebuffered saline (137 mM NaCl; $0.27 \mathrm{mM} \mathrm{KCl} ; 1 \mathrm{mM}$ phosphate buffer, $\mathrm{pH}$ 7.4) under vacuum followed by overnight incubation in fresh fixative at $4{ }^{\circ} \mathrm{C}$. Samples were either cut without prior embedding using a vibratome Series 1000 (40- to $50 \mu \mathrm{m}$-thick, TAAB, Aldermaston, UK), according to Inácio et al. (2018) or embedded in paraffin (VWR International, Radnor, Pennsylvania, USA) before sectioning. Before paraffin embedding, samples were dehydrated with a graded ethanol series $(50,70,85$, 95 and $100 \%$ ) and cleared with histoclear (VWR International). Tissue sections (7- $\mu \mathrm{m}$ thick) were made using a microtome (MEDITE M530, Burgdorf, Germany) and mounted on poly-Llysine pre-coated (1 $\mathrm{mg} \mathrm{ml}^{-1}$, Sigma-Aldrich, Madrid, Spain) slides.

\section{Histochemistry studies}

The vanillin- $\mathrm{HCl}$ assay was performed to estimate the presence of PA (Gardner 1975) in YDC and TDC vibratome sections. These were stained using a saturated alcoholic vanillin solution followed by the addition of concentrated $\mathrm{HCl}$ drops.

To identify the vacuoles and nuclei acids, YDC sections were stained with $0.1 \%$ acridine orange (Sigma-Aldrich) and observed under fluorescence. Acridine orange is a fluorescent basic dye that is lipid permeant, however in acidic conditions becomes protonated and trapped within vesicles (Correa et al. 2002). It can also intercalate double-stranded DNA with an excitation maximum at $502 \mathrm{~nm}$ and an emission maximum at
$525 \mathrm{~nm}$, and single-stranded RNA resulting in aggregates that emit in the red wavelength, with the excitation maximum at 640 nm (Darzynkiewicz 1990).

All preparations were observed on a Leitz Biomed microscope (Leica Microsystems, Wetzlar, Germany) and photographed with an AxionVision color camera (Carl Zeiss, Oberkochen, Germany). The observation of periderms with an Axio Imager.Z1 epifluorescence microscope (Carl Zeiss) using the Zeiss the filter set $49(445 / 50 \mathrm{~nm})$ allowed the identification of autofluorescence emitting tissues.

\section{Ultrastructure analysis}

For electron microscopy analysis, 2-year-old sprigs from different trees were cut in pieces of $\approx 0.3 \mathrm{~cm}$ long and fixed immediately after collection for $16 \mathrm{~h}$ in $5 \%$ glutaraldehyde in $0.08 \mathrm{M}$ PIPES buffer at $4{ }^{\circ} \mathrm{C}$. After washing $3 \times 5 \mathrm{~min}$ in $0.3 \mathrm{M}$ PIPES buffer at $4{ }^{\circ} \mathrm{C}$, vibratome sections with $100 \mu \mathrm{m}$ were made according to Inácio et al. (2018), and kept in the same fixative for at least $1 \mathrm{~h}$ at $4{ }^{\circ} \mathrm{C}$. Vibratome sections were washed in $0.3 \mathrm{M}$ PIPES buffer at $4{ }^{\circ} \mathrm{C}$ and post-fixed in $1 \%$ osmium tetroxide with $0.2 \mathrm{M}$ PIPES $1 \mathrm{~h}$ on ice (with agitation in the dark). After washing in distilled water, the sections were post-fixed and counterstained with uranyl acetate $1 \%$ in distilled water, for $30 \mathrm{~min}$. The sections were then dehydrated in a graded ethanol series (50, 70, 96 and 100\%) and pre-impregnated with a mixture of $1: 1100 \%$ ethanol, to avoid lipid loss during substitution and EPON Resin (Embed 812 kit with DMP-30, Electron Microscopy Sciences, Hatfield, Pennsylvania, USA) for $1 \mathrm{~h}$. The sections were then impregnated with $100 \%$ EPON resin overnight at $4{ }^{\circ} \mathrm{C}$ and flat embedded. For flat embedding, six-folds of parafilm were attached to each end of a glass slide and a square of plastic film (ACLAR ${ }^{\circledR}$ Fluoropolymer Films, Electron Microscopy Sciences) was placed on the slide between them. A fresh drop of room temperature EPON resin was placed onto the plastic film and the sections were dropped on it. A new square of plastic film was used to cover the sections, and a blank glass slide was laid on top. After polymerization at $60{ }^{\circ} \mathrm{C}$ for at least $48 \mathrm{~h}$ in a flat position, the glass slides were gently separated apart, and the plastic films were peeled off. The resin around the desired fragments was dissected with a razor blade, and the fragments were glued vertically onto a dummy blank resin block (only made of resin) with a drop of fresh room temperature resin. The blocks were inverted onto parafilm and placed in the oven at $60{ }^{\circ} \mathrm{C}$ for at least $24 \mathrm{~h}$. The parafilm was peeled off from the top of the block and the region of interest (ROI) was selected using a binocular scope attached to the ultramicrotome. The excess resin was trimmed, and the $\mathrm{ROI}$ sectioned with a histo diamond knife (Nidau, Switzerland) on a Reichert-Jung SuperNova ultramicrotome (Leica Microsystems). Ultra-thin sections of the ROI (80-90 nm) were collected into formvar-coated slot copper grids (Agar Scientific, Stansted, UK) contrasted with 
uranyl acetate/lead citrate, and screened under the electron microscope Hitachi H-7650 (Hitachi High-Technologies, Tokyo, Japan) at $100 \mathrm{kV}$.

\section{Immunolocalization of tonoplast intrinsic protein}

Immunodetection of tonoplast intrinsic protein 2 (TIP2) in YDC was performed according to Inácio et al. (2018). Young differentiating cork sections were incubated with primary antibody anti-TIP2;1,2;2,2;3 (1:100 dilution, AS12 2619, Agrisera, Vännäs, Sweden) and goat polyclonal secondary antibody to rabbit IgG-H\&L conjugated to Alexa Fluor ${ }^{\circledR} 488$ (1:200 dilution, AB150077, Abcam, Cambridge, UK). The slides were mounted in VECTASHIELD Mounting Medium with DAPI (Vector Laboratories, Burlingame, California, USA). A series of $z-$ stack with 0.5- $\mu \mathrm{m}$ intervals were acquired on Axio Imager.Z1 epifluorescence microscope (Carl Zeiss) using the Zeiss filter sets 49 for DAPI (excitation $365 \mathrm{~nm}$, beam splitter $395 \mathrm{~nm}$, and emission $445 / 50 \mathrm{~nm})$, and 44 for Alexa Fluor ${ }^{\circledR} 488$ (excitation $475 / 40 \mathrm{~nm}$, beam splitter $500 \mathrm{~nm}$, and emission $530 / 50 \mathrm{~nm}$ ), and projections of maximum fluorescence images were obtained.

\section{Characterization of cork oak putative proteins associated} with cork differentiation

The genes studied in this work are related to cell wall suberization: cork oak CYP86A32, previously characterized revealing high-sequence conservation between orthologous proteins and felling into the same clade (Serra et al. 2009), KCR1 and HCT; to the biosynthesis of condensed tannins: $L A R, L D O X$ and BAN; to condensed tannins transport: DTX41; to autophagy: ATG8F; and to PCD: $\gamma$ VPE, END04, PASPA3, RPN5A, XCP1, PR1 and AMC1.

The putative homologous of target sequences in cork oak were obtained by performing a BLASTp at cork oak ESTs database (http://corkoakdb.org) (Pereira-Leal et al. 2014) using Arabidopsis protein sequences retrieved from TAIR10 database (https://www.arabidopsis.org/) as a query. Since the Arabidopsis genome lacks the leucoanthocyanidin reductase (LAR) gene (Abrahams et al. 2003), the Desmodium uncinatum LAR ortholog protein was used (Tanner et al. 2003). The putative domain functional sites were predicted using the InterProScan platform (Zdobnov and Apweiler 2001). The potential cork oak orthologous proteins in other woody species were obtained by performing a BLAST at the NCBI database and Oak Genome (http://www.oakgenome.fr/). All sequences were aligned with MUSCLE (Edgar 2004), and the alignment was further trimmed with GBLOCKS (Castresana 2000). Distances between cork oak putative proteins and their orthologs were inferred by the maximum likelihood method based on the JTT matrix-based model using MEGA 7 software (Kumar et al. 2016) with 1000 bootstrap replicates. The accession numbers of the cork oak putative proteins and orthologous proteins and respective genes used in the analyses are listed in Table S1, available as Supplementary data at Tree Physiology Online.

qRT-PCR analysis

Young DC of 1- and 3-year-old sprigs, TDC and dedifferentiated actively growing calli obtained from cork oak leaves were used to total RNA extraction with the Spectrum ${ }^{\text {TM }}$ Plant Total RNA kit (Sigma-Aldrich) according to the instructions of the manufacturer except for some minor modifications described elsewhere (Inácio et al. 2018). Primers were designed using Primer Premier 5.0 software (Premier Biosoft International, Palo Alto, CA, USA) except for housekeeping genes (ACT, actin; $C A C s$, clathrin adaptor complexes medium subunit family protein, GAPDH; glyceraldehyde 3-phosphate dehydrogenase; EF$1 \alpha$, elongation factor 1 -alpha) that were chosen from a previous gene expression study in cork oak (Inácio et al. 2018). Target genes, $\mathrm{NCBI}$ sequence accession numbers, primer sequences and amplicon size are described in Table S2, available as Supplementary data at Tree Physiology Online.

The real-time qPCR was performed in 96-well white reaction plates (Bio-Rad), using an IQ5 Real-Time PCR (Bio-Rad, Hercules, CA, USA), based on at least three biological and three technical replicates. No template controls were also included in triplicate for each primer pair. The amplification efficiencies of all genes were estimated using the LinRegPCR quantitative PCR data analysis program (Ruijter et al. 2009) with the raw fluorescence data as input. The QsEF- $1 \alpha$ and QSACT genes were used as reference genes according to their higher stability calculated by the Normfinder algorithm (Andersen et al. 2004). Normalized relative quantities were obtained through the $\Delta \Delta \mathrm{CT}$ method using calli as a negative control sample (Livak and Schmittgen 2001). The fold-change values relative to calli mean gene expression were transformed into the log2 scale to perform a heatmap, drawn using the pheatmap function in the $R$ environment.

Statistical differences between gene relative expression were evaluated either by comparing YDC vs calli, TDC vs calli and YDC vs TDC (YDC and TDC) through Student's t-tests.

\section{Results}

Cork cell differentiation exhibits morphological features of programmed cell death

To characterize the differentiation process up to cork cell death, we analyzed the morphology of cork cells in YDC at the ultrastructural level along with fluorescence microscopy.

Cell walls of the newer cork cell layer already exhibited autofluorescence when excited with UV light, denoting suberin deposition, except for the most recently divided cell (Figure 1, arrow), as previously reported (Graça and Pereira 2004, Inácio et al. 2018). The comparison of cork cells with phellogen and phelloderm cells allowed the identification of 


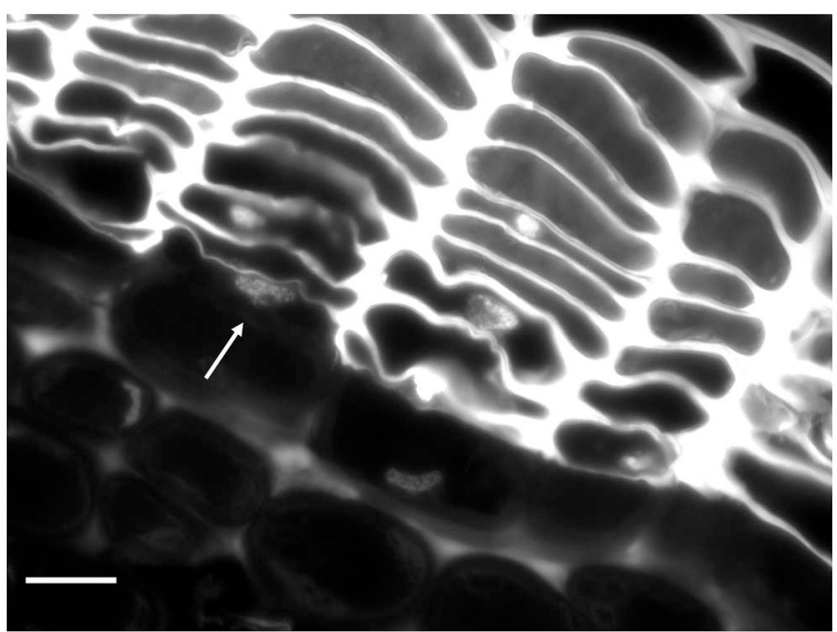

Figure 1. Cross section of the periderm of a 2-year-old cork oak sprig under UV light. Several cork cell layers show autofluorescence in their walls, except for the most recently formed cork cell (arrow). Bar $=10 \mu \mathrm{m}$.

cork cells PCD morphological features. While phellogen and phelloderm cells showed intact protoplasts occupying the whole cell, the youngest cork cells already showed some PCD characteristics (Figure 2A). Adjacent cork cells of the same layer presented distinct morphologies most probably related to the asynchrony of phellogen divisions (Figure 2A). The youngest cork cells (most recently divided and closer to the phellogen) showed more than one vacuole, surrounded by a dense cytoplasm (Figure 2B), autophagosome-like structures (Figure 2B, arrows), altered mitochondria (see Figure S3 available as Supplementary data at Tree Physiology Online) and electron-dense material (Figure 2C, white arrows) near the endoplasmic reticulum and inside the vacuole within vesicle-like structures (Figure $2 \mathrm{C}$, black arrow). The cells formed just before showed a large vacuole, a very thin cytoplasm layer (Figure 2D) with an apparent retraction (Figure 2D, arrow), and a continuous layer of electrondense material at the inner tonoplast surface also observed in phellogen cells (Figure 2A, arrow). Cork cells from the fourth layer showed complete vacuoles filling with the electron-dense material (Figure 2F). Cells from the fifth layer showed the entire cell lumen filled with the electron-dense deposits and no cytoplasm could be perceived (Figure 2G). The transition from electron-dense material filled cells to 'mature' cork cells with empty lumens was not detected in the ultrastructure of YDC, up to the second year of growth.

The analysis of cork cells nuclei from consecutive cell layers revealed apparently intact nuclei at the early stages of cork cells differentiation as detected by the green staining with acridine orange (Figure $2 \mathrm{H}$ ), whereas a shift to red at a later stage was observed (Figure 2l, arrow), suggesting DNA damage.
Vacuole membrane integrity was detected by immunodetection of tonoplast intrinsic proteins (TIP2;1, 2;2, 2;3 that showed the presence of vacuoles with integer tonoplast until the outermost cork cell layers, where the vacuole was not detected (Figure 3). Acridine orange-red staining also allowed to identify the acidic vacuoles (Figure $2 \mathrm{H}$ and I; Correa et al. 2002).

To investigate whether the electron-dense material found inside vacuoles was proanthocyanidins (PA), YDC was stained with vanillin- $\mathrm{HCl}$. This test results in red color in the presence of catechin, one of the building blocks of the proanthocyanidins (Gardner 1975). Cork cells approximately from the fifth layer up to the outermost layers, where the cells filled with electrondense material were found, were vanillin- $\mathrm{HCl}$ positive in the entire cell lumen (red color, Figure 4A), while the innermost layers show an absence of red.

The presence of PA was also investigated in TDC through vanillin- $\mathrm{HCl}$ test disclosing a distinct subcellular localization from YDC. Instead of the cell lumen, the cell walls were vanillin- $\mathrm{HCl}$ positive, with the color intensity substantially increasing from early differentiating cells (Figure 4B) to later cell differentiation stages (Figure 4C).

Differences in YDC and TDC cell size were also detected: YDC $\approx 16-32 \mu \mathrm{m}$ (inset in Figure 4A) and TDC $\approx 30-70 \mu \mathrm{m}$ (Figure 4B and $C$ ).

Genes related to suberin and proanthocyanidins biosynthesis, autophagy and programmed cell death were differentially expressed in young vs traumatic differentiating cork

The characterization of cork oak putative proteins associated with suberin biosynthesis, PA biosynthesis, autophagy and PCD revealed that they bear the conserved domains present in orthologous characterized proteins (see Table S3 available as Supplementary data at Tree Physiology Online). No cork oak homologous of the aspartic proteinase A3 (encoded by APA3 or PASPA3), which has been associated with plant $\mathrm{APCD}$ (Olvera-Carrillo et al. 2015, Wunderling et al. 2018) was found. The closest aspartic proteinase (QsPASPA) found and used in this study showed only $68 \%$ identity with PASPA3. The phylogenetic analysis generally grouped $Q$. suber proteins closely with orthologous proteins from other perennial tree species, particularly with the one from another Quercus species, Quercus robur (see Figure S4 available as Supplementary data at Tree Physiology Online).

The relative expression of genes related to suberin biosynthesis, proanthocyanidin biosynthesis and transport, autophagy and PCD were analyzed through GRT-PCR in YDC, TDC and calli, where suberin biosynthesis, PA biosynthesis, autophagy and PCD processes are presumably absent, and represented relative to calli tissue (Figure 5).

Genes related to suberin-QsKCR1, QsCYP86A32 and QsHCT - were expressed at significantly higher levels in both YDC $(P<0.0001, P<0.0001, P=0.0001$, respectively $)$ and 


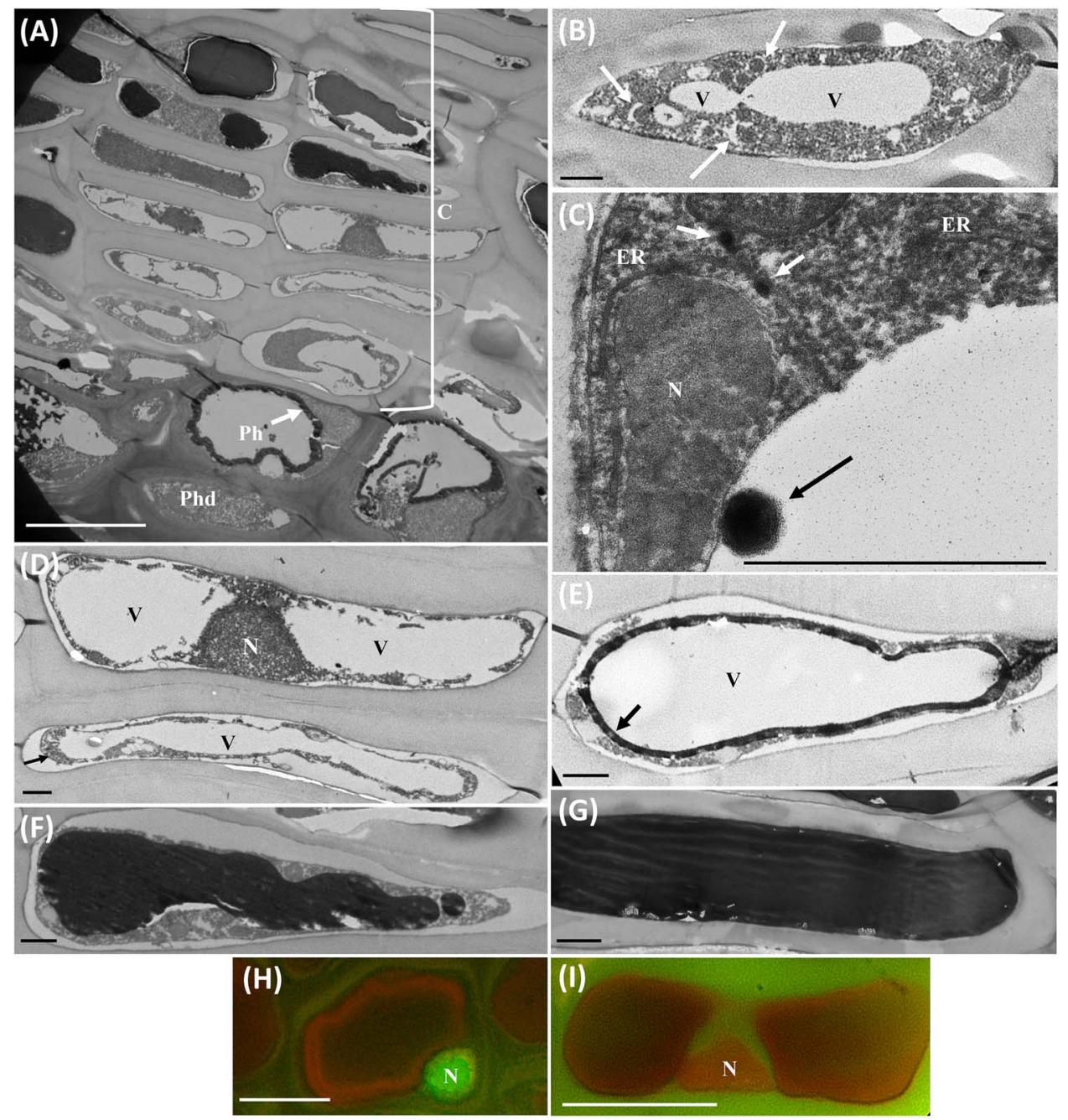

Figure 2. Cork cells PCD stages in a transverse section of a 2-year-old cork oak sprig. (A) Cork cells (c) ultrastructural changes compared with the phellogen mother-cell and phelloderm cells. (B) Cork cell at stage I of PCD showing two vacuoles, a dense cytoplasm and autophagosome-like structures (arrows). (C) Cork cell at stage II showing small vesicle-like structures with electron-dense material (arrows) close to the endoplasmic reticulum and inside the vacuole (arrow). (D) Cork cells at stage II exhibiting two or a large vacuole with an apparent retraction of the cytoplasm (arrow), and a thin cytoplasmic layer. (E) Cork cell at stage II presenting electron-dense deposits on the inner side of the tonoplast. (F) Cork cell at stage III displaying a vacuole filled with electron-dense material. $(G)$ Cork cell at stage IV showing the entire cell lumen filled with the electron-dense material. $(\mathrm{H})$ Cork cell at an early PCD stage showing a green nucleus with acridine orange stain. (I) Cork cell at a later PCD stage showing a red nucleus with acridine orange stain. $\mathrm{V}$, vacuole, $\mathrm{N}$, nucleus, $\mathrm{C}$, cork cells, Ph, phellogen cell, Phd, phelloderm cell, ER, endoplasmic reticulum. Bar in $\mathrm{A}=10 \mu \mathrm{m}$ and $\mathrm{B}-\mathrm{I}=1 \mu \mathrm{m}$.

TDC $(P<0.0001, P<0.0001, P<0.0001$, respectively $)$, when compared with calli. Moreover, a significantly higher accumulation of those transcripts was found in TDC when compared with YDC $(P<0.0001, P<0.0001, P<0.0001$, respectively). Genes related to PA—QsLDOX, Qs $L A R, Q s B A N$ and QsDTX41-were also expressed at significantly higher levels in both YDC $(P<0.0001, P=0.0002, P<0.0001$, $P=0.0001$, respectively $)$ and TDC $(P<0.0001, P<0.0001$, $P<0.0001, P<0.0001$, respectively) when compared with calli. A significantly higher accumulation of QsLDOX, QsLAR and QsDTX41 transcripts was found in TDC when compared with
YDC $(P=0.0004, P<0.0001, P=0.0002$, respectively $)$ while similar levels of $Q s B A N$ were detected $(P=0.6398)$.

The relative expression of QsPR1 and QSAMC1 genes, known to be induced during the hypersensitive cell death response (HR) and abiotic stress (Coll et al. 2010, Khan et al. 2015, Chandrashekar et al. 2018), was studied together with QsRPN5A, Qs VVPE, QsPASPA, QsENDO4, QsATG8F and QSXCP1 genes likely involved in APCD. QSAMC1 was downregulated in YDC when compared with calli $(P=0.0009)$ and not detected in TDC. Although the QsPR1 gene was detected at very low levels in YDC, no significant differences 


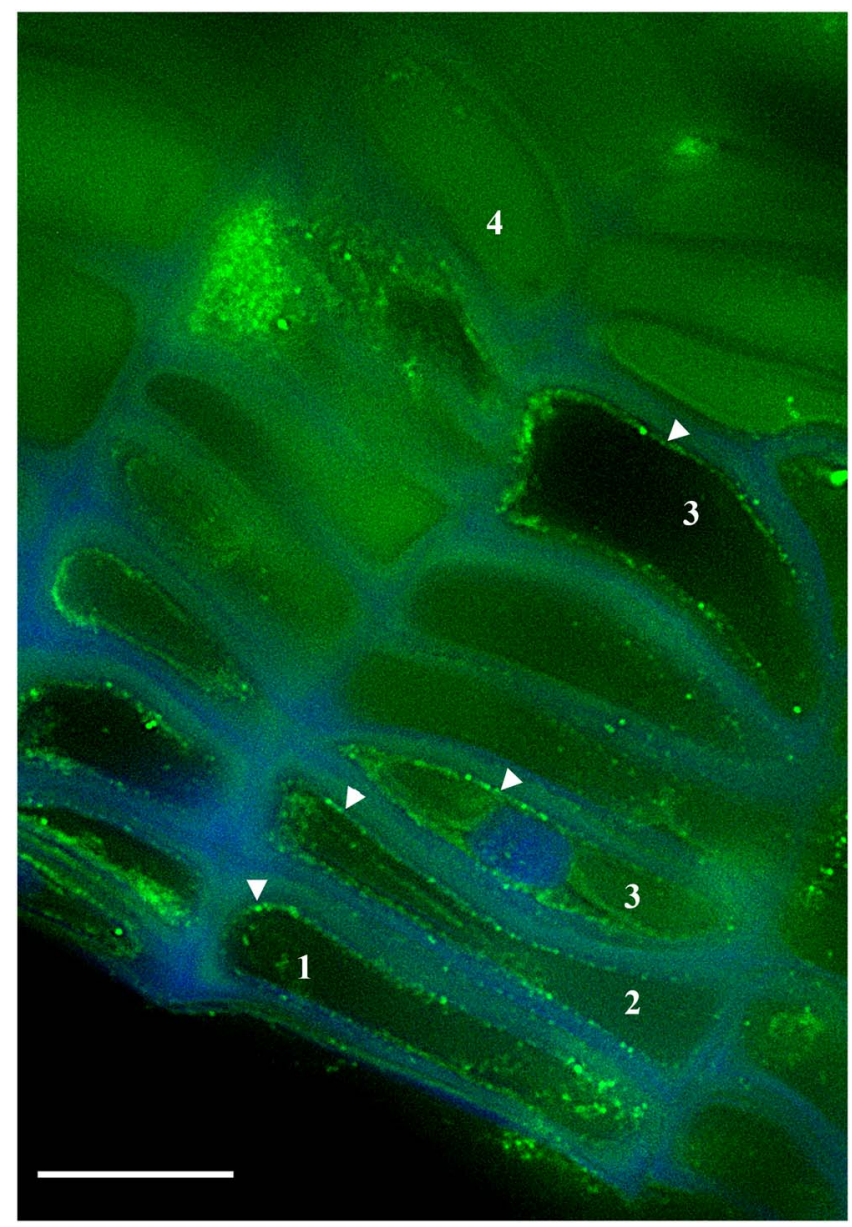

Figure 3. Immunolocalization of tonoplast intrinsic proteins TIP2;1, $2 ; 2,2 ; 3$ in cross sections of young sprigs shows integer tonoplast (arrowheads) at PCD stages I, II, III and its absence in IV. Bar $=10 \mu \mathrm{m}$.

were found when compared with calli $(P=0.3656)$. In TDC, the QsPR1 gene was significantly less expressed when compared with calli $(P=0.0208)$ and with YDC $(P=0.0284)$. In YDC, the relative expression of QSPASPA and QSATG8F genes was detected at a significantly higher level than in calli $(P=0.0011$, $P=0.0002$, respectively), whereas Qs $\gamma$ VPE, QsRPN5A and QsENDO4 were significantly less expressed $(P=0.0397$, $P=0.0383, P<0.0001$, respectively). In TDC, QsPASPA and QSATG8F were also upregulated when compared with calli $(P<0.0001, P<0.0001$, respectively) together with $Q s \gamma V P E$ and QSRPN5A ( $P=0.0001, P=0.0091$, respectively), whereas QsENDO4 was downregulated $(P<0.0001)$. QsPASPA, Qs $\gamma$ VPE, QsRPN5A and QsENDO4 genes were detected at significantly higher levels in TDC when compared with YDC $(P<0.0001, P<0.0001, P<0.0001, P=0.0041$, respectively), while the expression levels of QsATG8F were not significantly different $(P=0.2356)$. No detectable levels of expression were found for QsXCP1 in YDC, TDC and calli.

\section{Discussion}

The main objective of this work was to elucidate the cork differentiation process at the subcellular and molecular levels using cork oak as a periderm model plant. A combination of ultrastructural, histochemical and gene expression methods revealed a detailed set of subcellular morphological alterations, where the cell differentiation and death programs apparently initiate simultaneously. Differences between the first young and traumatic periderm differentiation were also highlighted.

Traumatic differentiation cork shows a higher rate of suberization than young differentiating cork

Cork cell walls are characterized by the deposition of suberin at the internal side of the cell wall forming an insulating matrix (reviewed in Graça 2015). We confirmed here a fast suberin deposition in recently divided cork cells before the next phellogen division in YDC (Figure 1), as already been described in young sprigs (Graça and Pereira 2004) and traumatic periderms (Inácio et al. 2018).

Among the main constituents specific of suberin are $\omega$ hydroxyacids, whose critical $\omega$-hydroxylation step synthesis is accomplished by the CYP86A1 enzyme (Hofer et al. 2008), while the chain elongation of very long-chain $\left(>C_{18}\right)$ suberin fatty acids (reviewed in (Franke et al. 2012) is promoted by the KCR1 enzyme (Beaudoin et al. 2009). Moreover, downregulating the early lignin pathway gene HCT dramatically decreases the levels of suberin-linked ferulic acid (Chen et al. 2006) denoting that HCT is also an important enzyme in suberin biosynthesis. Here, we observed the upregulation of QsCYP86A32, QsKCR1 and QsHCT in TDC when compared with YDC (Figure 5), which could indicate a higher rate of suberin deposition in the former. Indeed, traumatic phellogens formed after cork extraction and original phellogen death show an accelerated activity rate at 8 years of age, producing about 100 layers of cork cells per year (Pereira et al. 1992), in contrast to the original phellogens that produce only 3-6 layers in the first year of growth and up to 10 to 20 layers from the fifth to the seventh year (Graça and Pereira 2004). Similarly, the potato wound periderm exhibits an upregulation of suberinrelated genes (Vulavala et al. 2019) and down-regulation of $F H T$, a suberin feruloyl transferase, differentially affects native and wound periderms (Jin et al. 2018).

\section{Distinct cell localization of proanthocyanidins in young differentiating cork and traumatic differentiating cork}

Besides the similarity in terms of tissue origin, structure and morphology (Lapierre et al. 1996, Negrel et al. 1996, Schreiber et al. 2005), Q. suber virgin and amadia corks show variations in their chemical composition (Pereira 1988), also found in potato native and wound periderms. A striking difference between YDC and TDC concerns proanthocyanidins (PA) deposition since 

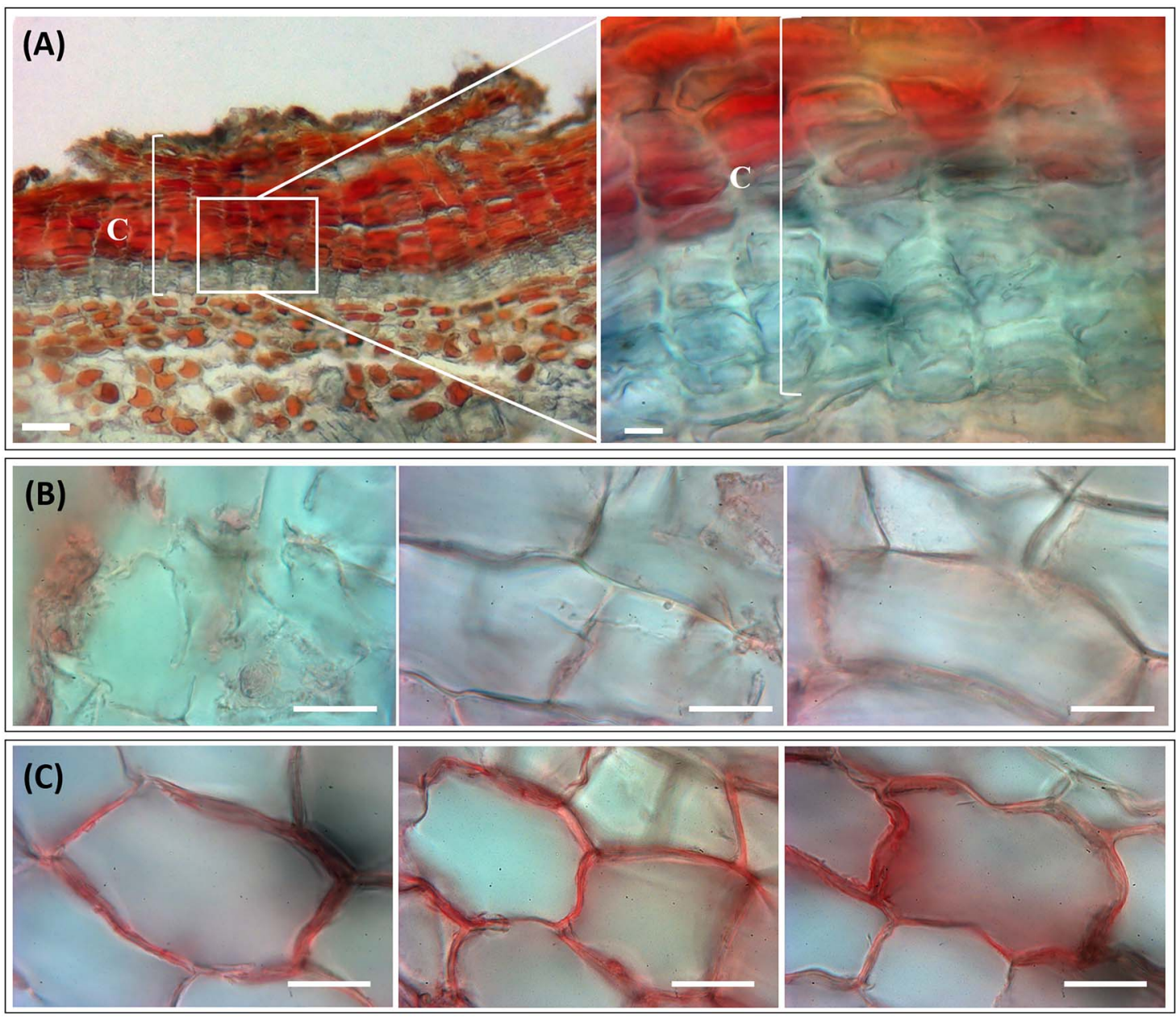

Figure 4. Proanthocyanidins detection by vanillin- $\mathrm{HCl}$ in 3-year-old cork oak periderms. (A) Cross section showing red coloration approximately from the fifth cork cell layer up to the outermost layers within the cell lumen (magnification). (B) Transverse section of a traumatic periderm showing a pale red coloration in the cell walls of cork cells at early differentiation stages. (C) Transverse section of a traumatic periderm showing an intense red coloration in the cell walls of cork cells at late differentiation stages. Bar in $A=50 \mu \mathrm{m}$, inset $=10 \mu \mathrm{m}, \mathrm{B}$ and $\mathrm{C}=20 \mu \mathrm{m}$.

these were found filling the cell lumen in YDC but appeared 'attached' to cell walls in TDC. Proanthocyanidins are built from flavan-3-ols, namely catechins, and their corresponding epi-stereoisomers are synthesized by the NADPH-dependent enzymes LAR, a leucoanthocyanidin reductase (Tanner et al. 2003), and BAN, an anthocyanidin reductase (Xie et al. 2003). Common steps are shared in the biosynthesis of epicatechin and anthocyanins where LDOX, a leucoanthocyanidin dioxygenase, is a key enzyme (Abrahams et al. 2003). Proanthocyanidins are synthesized in the endoplasmic reticulum and transported by vesicle trafficking and membrane transporters like the MATE protein 41 or DTX41 (reviewed in Zhao 2015) that mediates their vacuolar transport.

The presence of all the functional domains in the putative QsLAR, QsBAN, QsLDOX and QsDTX41 reported here supports the belief that their functions in cork oak are similar to the ones already described, namely the biosynthesis of PAs. Interestingly, the QsLAR, QsDTX41 and QsLDOX genes are overexpressed in TDC where PA have a low contribution to their total soluble phenolic content (Conde et al. 1998). However, this can be explained by the PA accumulation as part of cell walls in cork during traumatic periderm formation. In YDC, PA seem to appear in vesicle-like structures close to the endoplasmic reticulum and inside the vacuole near the tonoplast (Figure 2C). The upregulation of QSDTX41 in both YDC and TDC corroborates the possible involvement of the tonoplast in the differentiation of cork oak periderm cells; however, in the case of TDC, these flavonoids may also be transported to the apoplast through this transporter coupled with vesicle trafficking (Zhao 2015).

Proanthocyanidins are usually sequestered in compartments like the vacuole due to their toxicity to the plant cell (Shimada et al. 2018), but can also be found in cell walls where the PA polymerization level is higher than in the internal part of the cell (Geny et al. 2003). The gradual PA associated-color intensity seen in traumatic cell walls suggested that PAs are more polymerized in cell walls as cells differentiate since the color 


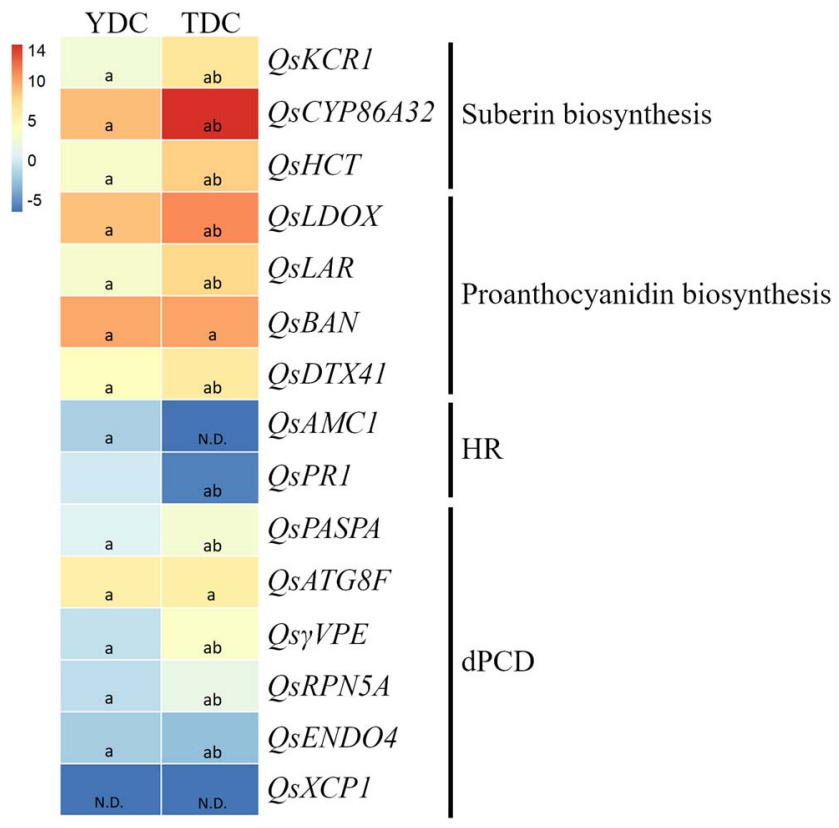

Figure 5. Heatmap representing the mean log2 fold change in gene expression in young differentiating cork (YDC) and traumatic differentiating cork (TDC) relative to calli tissue and evaluated by qRT-PCR. The expression levels were normalized with the geometric mean of reference genes QsEF-1 $\alpha$ and QsACT. Letter 'a' indicates significant differences $(P<0.0397)$ between YDC or TDC and calli and letter ' $b$ ' indicates significant differences $(P<0.0284)$ between YDC and TDC, tested through Student's $t$-tests. N.D., no detectable levels of expression.

yield is lower for catechin-units than for the polymer (Price et al. 1978). Taking into account the essential roles of PA polymertype flavonoids in plant protection against microbial pathogens and insect pests (Saddique et al. 2018), we hypothesize that PA accumulation seen in YDC might also be a defense mechanism against pathogenic attack (Tiberi et al. 2016). In TDC, where PA seem to be part of the cell wall presumably in association with suberin, they would play the same protective role against biotic aggression in vacuole-less cells. Indeed, cork from $Q$. suber is exceptional in the fact it does not rot (DeBaun and Nord 1951), even when exposed in the environment for a very long time.

\section{Young differentiating cork is characterized by distinct programmed cell death stages}

The final step in cork cells differentiation is their death, commanded by a strict developmental PCD. Plant PCD is a common process present in several organs and stages of development, although there is no consensus regarding the types undergoing in plants. Some authors argue that only 'autolytic' or 'nonautolytic' types exist, with the main difference residing on the cytoplasm clearance quickness after the tonoplast rupture in the former or the increased tonoplast permeability in the latter (van Doorn 2011), whereas other authors propose that an apoptosis-like process exists in plants (reviewed in Dickman et al. 2017).
Although empty cells could not be observed in YDC up to 3 years old studied here, initial PCD features were detected just after phellogen division (Figure 2A). This suggests that the PCD program initiates simultaneously with the initial steps of cell differentiation like the rapid cell wall suberization (Graça and Pereira 2004, Inácio et al. 2018), in contrast with developing tracheary elements, where PCD is initiated by the rupture of the central vacuole (reviewed in Fukuda 2016). The differences in cell size between YDC and TDC (Figure 4) suggest that the most recently formed cells do not yet have the definitive size, still expanding until complete suberization of their walls. Following the fast suberization of the cell wall, we were able to define distinct stages of cork cells differentiation and PCD for the first time, according to cork cells morphology: stage I—cells with several vacuoles, dense cytoplasm, autophagosome-like structures, altered mitochondria and small deposits of electrondense material (Figure 2B); stage II—cells with a large vacuole, thin cytoplasm layer, apparent retraction of the cytoplasm and a continuous layer of electron-dense material at the inner tonoplast surface (Figure 2D); stage III-cells with a vacuole filled with electron-dense material (Figure 2F); and stage IV-cells with the entire lumen filled with electron-dense material and no observed cytoplasm (Figure 2G). The boundary between stage III and stage IV seems to implicate the tonoplast rupture as the entire cell lumen was seen filled with electron-dense deposits and no cytoplasm could be perceived. The presence of a large vacuole in cells from stage II onwards suggests that the small vacuoles merge into one, resulting in a decrease of cytoplasm volume, as already proposed in several types of autolytic PCD (van Doorn 2011). These changes in the morphology of cork cells along with the presence of autophagosome-like structures in the cytoplasm, DNA degradation and apparent tonoplast rupture are typical features of the proposed 'autolytic' type PCD (van Doorn 2011). Nevertheless, cork cells also exhibited features considered essential to the cascade of events that have been associated with an apoptotic-like PCD (reviewed in Dickman et al. 2017) such as the apparent protoplasm retraction involving the detachment of the plasma membrane of the cell wall and the nucleus condensation (Inácio et al. 2018). This combination of both autolytic and apoptotic-like hallmarks has also been observed in the self-incompatibility and hypersensitive responses (van Doorn 2011).

\section{Developmental programmed cell death occurs in young and traumatic differentiating cork}

To evaluate the involvement of well-characterized genes in cork differentiation and PCD, gene expression analysis was performed in both YDC and TDC, using calli as a control, since the studied processes are likely not active in this tissue. The differentiation of cork cells is characterized by the expression of autophagy and $\mathrm{DPCD}$-associated genes, particularly by the increase of QSATG8F, QsPASPA and Qs VVPE transcripts, and, 
in contrast, by the low levels of QSAMC1 and QsPR1 gene expression associated with abiotic/biotic stress (Khan et al. 2015). This suggests that dPCD rather than environmentally induced PCD is involved in cork oak periderm differentiation (reviewed in Daneva et al. 2016, Wunderling et al. 2018).

The upregulation of QsATG8F in both YDC and TDC agrees with the presence of the autophagosome-like structures and the possible role of autophagy in cork cells PCD. Developmental PCD processes involved in embryogenesis, male reproductive development or leaf senescence has also been associated with autophagy, which is an intracellular self-eating process involving the delivery of cytosolic components to the vacuole for their degradation and recycling (reviewed in Bozhkov 2018). ATG8 proteins are involved in autophagosome formation and are essential for autophagic membrane elongation (Shpilka et al. 2011). The ATG8F is considered a marker for autophagy and mediates the autophagosome delivery to the vacuole (Merkulova et al. 2014). Other PCD programs including xTE and fiber differentiation have shown an upregulation of autophagy-related genes (Courtois-Moreau et al. 2009, Kwon et al. 2010a).

Typical PCD features were recently observed at the chromatin level in periderms from 1- to 3-year-old cork oak sprigs, namely a progressive nuclear condensation with chromatin accumulation at the nuclear periphery and an increase in repressive chromatin marks (Inácio et al. 2018). The shift in acridine orange staining from an early to a later stage of cork cells differentiation suggests that other nuclear alterations such as DNA damage may occur. The same has been observed in wheat cells undergoing PCD where a progressive DNA fragmentation was correlated to chromatin condensation (Domínguez and Cejudo 2006). This contrasts with xylem xTE PCD, where no chromatin condensation is observed and the digestion of the nucleus is triggered by the tonoplast rupture (Obara et al. 2001). Considering that the tonoplast appears to be intact in PA-filled vacuoles until stage III, the major DNA degradation should take place by vacuolar collapse after the release of hydrolytic enzymes such as proteases and nucleases at stage IV. However, this disagrees with the unexpected downregulation of QsENDO4 in both YDC and TDC. This protein is a member of the S1-like nuclease family known to digest DNA (Lesniewicz et al. 2013) and being up-regulated during onion skin PCD (Galsurker et al. 2017). This finding indicates that other nucleases rather than QsENDO4 may be responsible for nuclear DNA degradation in cork cells. Another hypothesis is the active role of PA in DNA degradation since it is known that these polyphenols can induce apoptosis in cancer cells through nuclear condensation and DNA fragmentation (Rao and Pagidas 2010). $\gamma$ VPE activity has been found in the lytic vacuoles of vegetative tissues during senescence and under abiotic stress (Kinoshita et al. 1999), and VPEs seem to mediate the vacuolar collapse as VPE-silenced plants did not undergo tonoplast rupture or cell death (Hatsugai et al. 2004). Although low levels of expression found were for Qs $\gamma$ VPE in YDC, the significantly higher levels found in TDC, as already reported in virgin corks (Soler et al. 2007, Boher et al. 2018), suggest that tonoplast rupture does occur, probably during cork cells PCD from stages III to IV.

Even though the xylem development shares some similarities with periderm formation discussed above, the xylem cysteine protease XCP1 involved in the autolysis of XTE in Arabidopsis roots (Avci et al. 2008) was not expressed in both YDC and TDC, suggesting that other proteases might be involved in cork cell differentiation and PCD.

Despite the PCD features observed, the YDC is mainly composed of still-living cells (Inácio et al. 2018), which was corroborated by the significantly lower expression of cell deathrelated genes when compared with traumatic periderms. Indeed, the most significant increase in the levels of DNA methylation perhaps associated with the PCD process was found in cork cells in traumatic periderms (Inácio et al. 2018). Moreover, most of the studied genes were significantly upregulated in traumatic periderms when compared with the first young periderms. Different developmental stages associated with distinct mechanical tensions can also contribute to the observed differences. Secondary growth in young sprigs with small diameter implies a higher rate of anticlinal divisions of the vascular cambium and also of the phellogen comparing with larger stems producing traumatic periderms, where tangential tensions are lower. These results also suggest a faster metabolism and cell death process in traumatic compared with the first young periderms. This emphasizes the impact of the wounding on the phellogen activity, leading to the production of more phellogen-derivatives during the growth season, revealing its quick ability to raise a new protective tissue after suffering a dramatic aggression ensuring the viability of the tree, as recently suggested in other works (Lopes et al. 2020). This functioning highlights the tremendous adaptive capacity of cork oak to cope with adverse conditions.

\section{Conclusion}

Cork is a fundamental protective tissue in plants with secondary growth, and a unique forest product that constitutes the basis of a sustainable and profitable industry, compatible with other land uses like agriculture and livestock. This work is, to our knowledge, the first systematic study focusing on the distinct processes underlying cork formation at the cellular, ultrastructural and molecular levels using cork oak periderm as a model system.

The approaches used to follow the periderm development from the first-formed young tissue to the well-developed raw cork used in the industry enabled a comprehensive overview of the distinct processes underlying cork formation, thus significantly contributing to the disclosure of the cork ontogenic 
program. We confirmed that marker genes of suberin and proanthocyanidin biosynthesis, autophagy and PCD are simultaneously expressed in differentiating cork tissue, corroborating the morphological and histochemical analysis. To decipher the exact role of the studied genes and other genes putatively involved in signaling, regulation and execution of cork differentiation and PCD, functional studies must be conducted.

This work thus provides insights into a fundamental protective structure of the plant secondary body, as well as into a unique product with sustainable and profitable use, the cork.

\section{Supplementary data}

Supplementary data for this article are available at Tree Physiology Online.

\section{Acknowledgments}

We are very grateful to Fundação João Lopes Fernandes for giving access to the Herdade dos Leitões trees and providing the best conditions for cork harvesting. We also thank the Electron Microscopy Service, part of the Histology and Comparative Pathology Laboratory, Instituto de Medicina Molecular, for the transmission electron microscopy analysis. We are very thankful to Pedro Barros (ITQB, Nova University of Lisbon) for kindly provide the calli obtained from $Q$. suber leaves. We also thank the two reviewers for the helpful suggestions and comments that significantly contribute to improve the manuscript. Our thanks are also due to Forest Research Centre (CEF, UID/AGR/00239/2013).

\section{Conflict of interest}

None declared.

\section{Funding}

This work was supported by the Portuguese Foundation for Science and Technology (FCT) (http://www.fct.pt/) through the project PTDC/AGR-FOR/3356/2014, V.I.'s PhD grant (PhD grant SFRH/BD/85879/2012) and research unit Linking Landscape, Environment, Agriculture and Food (LEAF) (UID/AGR/04129/2013 and UID/AGR/04129/2020).

\section{Authors' contributions}

V.I. performed all the experiments, data analysis and interpretation, and worked on the original draft of the manuscript. C.L. helped in vanillin-HCl tests. J.G. conceived the tree sampling, analyzed the data and critically reviewed the manuscript. L.M.C. conceived and designed the experiments, analyzed the data and critically reviewed the manuscript.

\section{References}

Abrahams S, Lee E, Walker AR, Tanner GJ, Larkin PJ, Ashton AR (2003) The Arabidopsis TDS4 gene encodes leucoanthocyanidin dioxygenase (LDOX) and is essential for proanthocyanidin synthesis and vacuole development. Plant J 35:624-636.

Alonso-Serra J, Safronov O, Lim K et al. (2019) Tissue-specific study across the stem reveals the chemistry and transcriptome dynamics of birch bark. New Phytol 222:1816-1831.

Andersen CL, Jensen LJ, Ørntoft TF (2004) Normalization of real-time quantitative reverse transcription-PCR data: a model-based variance estimation approach to identify genes suited for normalization, applied to bladder and colon cancer data sets normalization of real-time quantitative reverse. Cancer Res 64:5245-5250.

Arzee T, Liphschitz N, Waisel Y (1968) The origin and development of the Phellogen in Robinia pseudacacia L. New Phytol 67:87-93.

Arzee T, Waisel Y, Liphschitz N (1970) Periderm development and phellogen activity in the shoots of Acacia raddiana Savi. New Phytol 69:395-398.

Arzee T, Arbel E, Cohen L (1977) Ontogeny of periderm and phellogen activity in Ceratonia siliqua L. Bot Gaz 138:329-333.

Avci U, Earl Petzold H, Ismail IO, Beers EP, Haigler CH (2008) Cysteine proteases XCP1 and XCP2 aid micro-autolysis within the intact central vacuole during xylogenesis in Arabidopsis roots. Plant J 56: 303-315.

Beaudoin F, Wu X, Li F, Haslam RP, Markham JE, Zheng H, Napier $J A$, Kunst $L$ (2009) Functional characterization of the Arabidopsis $\beta$ ketoacyl-coenzyme A reductase candidates of the fatty acid elongase. Plant Physiol 150:1174-1191.

Biggs AR (1986) Phellogen regeneration in injured peach tree bark. Ann Bot 57:463-470.

Biggs AR, Northover $J$ (1985) Formation of the primary protective layer and phellogen after leaf abscission in peach. Can J Bot 63:1547-1550.

Boher P, Soler M, Sánchez A, Hoede C, Noirot C, Paiva JAP, Serra O, Figueras M (2018) A comparative transcriptomic approach to understanding the formation of cork. Plant Mol Biol 96:103-118.

Bonneau L, Ge Y, Drury GE, Gallois P (2008) What happened to plant caspases? J Exp Bot 59:491-499.

Bozhkov PV (2018) Plant autophagy: mechanisms and functions. J Exp Bot 69:1281-1285.

Brillouet J-M (2014) Plasticity of the tannosome ontogenesis in the Tracheophyta. J Plant Sci 2:317-323.

Capote T, Barbosa P, Usié A, Ramos AM, Inácio V, Ordás R, Gonçalves S, Morais-Cecílio L (2018) ChIP-Seq reveals that QsMYB1 directly targets genes involved in lignin and suberin biosynthesis pathways in cork oak (Quercus suber). BMC Plant Biol 18:198.

Castresana J (2000) Selection of conserved blocks from multiple alignments for their use in phylogenetic analysis. Mol Biol Evol 17:540-552.

Chandrashekar N, Ali S, Grover A (2018) Exploring expression patterns of PR-1, PR-2, PR-3, and PR-12 like genes in Arabidopsis thaliana upon Alternaria brassicae inoculation. 3 Biotech 8:1-10.

Chen F, Srinivasa Reddy MS, Temple S, Jackson L, Shadle G, Dixon RA (2006) Multi-site genetic modulation of monolignol biosynthesis suggests new routes for formation of syringyl lignin and wall-bound ferulic acid in alfalfa (Medicago sativa L.). Plant J 48 113-124.

Coll NS, Vercammen D, Smidler A, Clover C, Van Breusegem F, Dangl JL, Epple P (2010) Arabidopsis type I Metacaspases control cell death. Science 330:1393-1397.

Conde E, Cadahía E, García-Vallejo MC, De Simón BF (1998) Polyphenolic composition of Quercus suber Cork from different Spanish provenances. J Agric Food Chem 46:3166-3171. 
Correa NM, Biasutti MA, Silber JJ (2002) Acid-Base and aggregation processes of acridine orange base in n-heptane/AOT/water reverse micelles. Langmuir 18:2039-2047.

Courtois-Moreau CL, Pesquet E, Sjödin A, Muñiz L, Bollhöner B, Kaneda M, Samuels L, Jansson S, Tuominen H (2009) A unique program for cell death in xylem fibers of Populus stem. Plant J 58: 260-274.

Daneva A, Gao Z, Van Durme M, Nowack MK (2016) Functions and regulation of programmed cell death in plant development. Annu Rev Cell Dev Biol 32:441-468.

Darzynkiewicz Z (1990) Differential staining of DNA and RNA in intact cells and isolated cell nuclei with acridine orange. In: Darzynkiewicz Z, Crissman H (eds) Methods in cell biology. Academic Press, Cambridge, Massachusetts, pp 285-298.

DeBaun RM, Nord FF (1951) The resistance of cork to decay by wooddestroying molds. Arch Biochem Biophys 33:314-319.

Dickman M, Williams B, Li Y, de Figueiredo P, Wolpert T (2017) Reassessing apoptosis in plants. Nat Plants 3:773.

Domínguez F, Cejudo FJ (2006) Identification of a nuclear-localized nuclease from wheat cells undergoing programmed cell death that is able to trigger DNA fragmentation and apoptotic morphology on nuclei from human cells. Biochem J 397:529-536.

van Doorn WG (2011) Classes of programmed cell death in plants, compared to those in animals. J Exp Bot 62:4749-4761.

Edgar RC (2004) MUSCLE: multiple sequence alignment with improved accuracy and speed. In: IEEE Computational Systems Bioinformatics Conference (CSB 2004). IEEE, New Jersey, EUA, pp 728-729.

Evert RF (2006) Esau's plant anatomy: meristems, cells, and tissues of the plant body: their structure, function, and development, 3rd edn. John Wiley \& Sons, Inc., New Jersey, EUA.

Franke RB, Dombrink I, Schreiber L (2012) Suberin goes genomics: use of a short living plant to investigate a long lasting polymer. Front Plant Sci 3:4

Fukuda H (2016) Signaling, transcriptional regulation, and asynchronous pattern formation governing plant xylem development. Proc Japan Acad Ser B 92:98-107.

Galsurker O, Doron-Faigenboim A, Teper-Bamnolker P, Daus A, Fridman Y, Lers A, Eshel D (2017) Cellular and molecular changes associated with onion skin formation suggest involvement of programmed cell death. Front Plant Sci 7:2031.

Gardner RO (1975) Vanillin-hydrochloric acid as a histochemical test for tannin. Stain Technol 50:315-317.

Geny L, Saucier C, Bracco S, Daviaud F, Glories Y (2003) Composition and cellular localization of tannins in grape seeds during maturation. J Agric Food Chem 51:8051-8054.

Graça J (2015) Suberin: the biopolyester at the frontier of plants. Front Chem 3:62.

Graça J, Pereira H (2004) The periderm development in Quercus suber. IAWA J 25:325-335.

Gričar J, Jagodic Š, Prislan P (2015) Structure and subsequent seasonal changes in the bark of sessile oak (Quercus petraea). Trees 29:747-757.

Hassanpour S, MaheriSis N, Eshratkhah B (2011) Plants and secondary metabolites (tannins): a review. Int J For Soil Eros 1:47-53.

Hatsugai N, Kuroyanagi M, Yamada K, Meshi T, Tsuda S, Kondo M, Nishimura M, Hara-Nishimura I (2004) A plant vacuolar protease, VPE, mediates virus-induced hypersensitive cell death. Science 305:855-858.

Hofer R, Briesen I, Beck M, Pinot F, Schreiber L, Franke R (2008) The Arabidopsis cytochrome P450 CYP86A1 encodes a fatty acid -hydroxylase involved in suberin monomer biosynthesis. J Exp Bot 59:2347-2360.

Inácio V, Martins MT, Graça J, Morais-Cecilio L (2018) Cork oak young and traumatic periderms show PCD typical chromatin patterns but different chromatin-modifying genes expression. Front Plant Sci 9:1194.

Jin L, Cai Q, Huang W, Dastmalchi K, Rigau J, Molinas M, Figueras M, Serra O, Stark RE (2018) Potato native and wound periderms are differently affected by down-regulation of FHT, a suberin feruloyl transferase. Phytochemistry 147:30-48.

Khan MIR, Fatma M, Per TS, Anjum NA, Khan NA (2015) Salicylic acidinduced abiotic stress tolerance and underlying mechanisms in plants. Front Plant Sci 6:462.

Kinoshita T, Yamada K, Hiraiwa N, Kondo M, Nishimura M, HaraNishimura I (1999) Vacuolar processing enzyme is up-regulated in the lytic vacuoles of vegetative tissues during senescence and under various stressed conditions. Plant J 19:43-53.

Kumar S, Stecher G, Tamura K (2016) MEGA7: molecular evolutionary genetics analysis version 7.0 for bigger datasets. Mol Biol Evol 33:1870-1874.

Kwon SI, Cho HJ, Jung JH, Yoshimoto K, Shirasu K, Park OK (2010a) The Rab GTPase RabG3b functions in autophagy and contributes to tracheary element differentiation in Arabidopsis. Plant J 64:151-164.

Kwon SI, Cho HJ, Park OK (2010b) Role of Arabidopsis RabG3b and autophagy in tracheary element differentiation. Autophagy 6:1187-1189.

Lapierre C, Pollet B, Négrel J (1996) The phenolic domain of potato suberin: structural comparison with lignins. Phytochemistry 42:949-953.

Lesniewicz K, Karlowski WM, Pienkowska JR, Krzywkowski P, Poreba E (2013) The plant S1-like nuclease family has evolved a highly diverse range of catalytic capabilities. Plant Cell Physiol 54: 1064-1078.

Livak KJ, Schmittgen TD (2001) Analysis of relative gene expression data using real-time quantitative PCR and the 2(-Delta Delta $C(T)$ ) method. Methods 25:402-408.

Lopes ST, Sobral D, Costa B, Perdiguero P, Chaves I, Costa A, Migue CM (2020) Phellem versus xylem: genome-wide transcriptomic analysis reveals novel regulators of cork formation in Cork oak. Tree Physiol 40:129-141.

Merkulova EA, Guiboileau A, Naya L, Masclaux-Daubresse C, Yoshimoto K (2014) Assessment and optimization of autophagy monitoring methods in arabidopsis roots indicate direct fusion of autophagosomes with vacuoles. Plant Cell Physiol 55:715-726.

Mogensen HL (1968) Studies on the bark of the Cork bark fir: Abies lasiocarpa var. Arizonica (Merriam) Lemmon I. Periderm ontogeny. J Arizona Acad Sci 5:36-40.

Negrel J, Pollet B, Lapierre C (1996) Ether-linked ferulic acid amides in natural and wound periderms of potato tuber. Phytochemistry 43:1195-1199.

Obara K, Kuriyama H, Fukuda H (2001) Direct evidence of active and rapid nuclear degradation triggered by vacuole rupture during programmed cell death in Zinnia. Plant Physiol 125:615-626.

Olvera-Carrillo Y, Van Bel M, Van Hautegem T et al. (2015) A conserved core of PCD indicator genes discriminates developmentally and environmentally induced programmed cell death in plants. Plant Physiol 169:2684-2699.

Oven P, Torelli N, Shortle WC, Zupančič M (1999) The formation of a ligno-suberised layer and necrophylactic periderm in beech bark (Fagus sylvatica L.). Flora 194:137-144.

Pereira H (1988) Chemical composition and variability of cork from Quercus suber L. Wood Sci Technol 22:211-218.

Pereira H, Graça J, Baptista C (1992) The effect of growth rate on the structure and compressive properties of cork. IAWA Bull 13:389-396.

Pereira-Leal JB, Abreu IA, Alabaça CS et al. (2014) A comprehensive assessment of the transcriptome of Cork oak (Quercus suber) through EST sequencing. BMC Genomics 15:371. 
Pinheiro C, Wienkoop S, de Almeida JF et al. (2019) Phellem cell-wall components are discriminants of cork quality in Quercus suber. Front Plant Sci 10:944.

Price ML, Van Scoyoc S, Butler LG (1978) A critical evaluation of the vanillin reaction as an assay for tannin in sorghum grain. J Agric Food Chem 26:1214-1218.

Rantong G, Gunawardena AHLAN (2015) Programmed cell death: genes involved in signaling, regulation, and execution in plants and animals. Botany 93:193-210.

Rao SD, Pagidas K (2010) Epigallocatechin-3-gallate, a natural polyphenol, inhibits cell proliferation and induces apoptosis in human ovarian cancer cells. Anticancer Res 30:2519-2523.

Rittinger PA, Biggs AR, Peirson DR (1987) Histochemistry of lignin and suberin deposition in boundary layers formed after wounding in various plant species and organs. Can J Bot 65:1886-1892.

Ruijter JM, Ramakers C, Hoogaars WMH, Karlen Y, Bakker O, Van den Hoff MJB, Moorman AFM (2009) Amplification efficiency: linking baseline and bias in the analysis of quantitative PCR data. Nucleic Acids Res 37:e45-e45.

Saddique M, Kamran M, Shahbaz M (2018) Differential responses of plants to biotic stress and the role of metabolites. In: Plant metabolites and regulation under environmental stress. Academic Press, Cambridge, Massachusetts, pp 69-87.

Saito K, Yonekura-Sakakibara K, Nakabayashi R, Higashi Y, Yamazaki M, Tohge T, Fernie AR (2013) The flavonoid biosynthetic pathway in Arabidopsis: structural and genetic diversity. Plant Physiol Biochem 72:21-34.

Schreiber L, Franke R, Hartmann K (2005) Wax and suberin development of native and wound periderm of potato (Solanum tuberosum L.) and its relation to peridermal transpiration. Planta 220:520-530.

Serra O, Soler M, Hohn C, et al. (2009) CYP86A33-targeted gene silencing in potato tuber alters suberin composition, distorts suberin lamellae, and impairs the periderm's water barrier function. Plant Physiol 149:1050-1060.

Shibui H, Sano Y (2018) Structure and formation of phellem of Betula maximowicziana. IAWA J 39:18-36.
Shimada T, Takagi J, Ichino T, Shirakawa M, Hara-Nishimura I (2018) Plant vacuoles. Annu Rev Plant Biol 69:123-145.

Shpilka T, Weidberg H, Pietrokovski S, Elazar Z (2011) Atg8: an autophagy-related ubiquitin-like protein family. Genome Biol 12:226.

Soler M, Serra O, Molinas M, Huguet G, Fluch S, Figueras M (2007) A genomic approach to suberin biosynthesis and cork differentiation. Plant Physiol 144:419-431.

Takanashi K, Shitan N, Yazaki K (2014) The multidrug and toxic compound extrusion (MATE) family in plants. Plant Biotechnol 31:417-430.

Tanner GJ, Francki KT, Abrahams S, Watson JM, Larkin PJ, Ashton AR (2003) Proanthocyanidin biosynthesis in plants purification of legume leucoanthocyanidin reductase and molecular cloning of its cDNA. J Biol Chem 278:31647-31656.

Tiberi R, Branco M, Bracalini M, Croci F, Panzavolta T (2016) Cork oak pests: a review of insect damage and management. Ann For Sci 73:219-232.

Vishwanath SJ, Delude C, Domergue F, Rowland O (2015) Suberin: biosynthesis, regulation, and polymer assembly of a protective extracellular barrier. Plant Cell Rep 34:573-586.

Vulavala VKR, Fogelman E, Faigenboim A, Shoseyov O, Ginzberg I (2019) The transcriptome of potato tuber phellogen reveals cellular functions of cork cambium and genes involved in periderm formation and maturation. Sci Rep 9:10216.

Waisel Y, Liphschitz N, Arzee T (1967) Phellogen activity in Robinia pseudacacia L. New Phytol 66:331-335.

Wunderling A, Ripper D, Barra-Jimenez A, Mahn S, Sajak K, Ben TM, Ragni $L$ (2018) A molecular framework to study periderm formation in Arabidopsis. New Phytol 219:216-229.

Xie D-Y, Sharma SB, Paiva NL, Ferreira D, Dixon RA (2003) Role of anthocyanidin reductase, encoded by BANYULS in plant flavonoid biosynthesis. Science 299:396-399.

Zdobnov EM, Apweiler R (2001) InterProScan-an integration platform for the signature-recognition methods in InterPro. Bioinformatics 17:847-848.

Zhao J (2015) Flavonoid transport mechanisms: how to go, and with whom. Trends Plant Sci 20:576-585. 